\title{
Diurnal Changes of Heart Rate and Sympathovagal Activity for Temporal Patterns of Transient Ischemic Episodes in 24-Hour Electrocardiograms
}

\author{
A. Smrdel and F. Jager \\ Faculty of Computer and Information Science, University of Ljubljana, 1000 Ljubljana, Slovenia
}

Received 26 April 2006; Revised 27 October 2006; Accepted 11 January 2007

Recommended by Maurice Cohen

We test the hypothesis that different temporal patterns of transient ST segment changes compatible with ischemia (ischemic episodes) are a result of different physiologic mechanisms responsible for ischemia. We tested the hypothesis using records of the Long-Term ST Database. Each record was divided into three intervals of records: morning, day, and night intervals; and was inserted into one of three sets according to the temporal pattern of ischemia: salvo, periodic, and sporadic pattern. We derived timeand frequency-domain parameters of the heart rate time series in selected intervals in the neighborhood of ischemic episodes. We used the adaptive autoregressive method with a recursive least-square algorithm for consistent spectral tracking of heart rate time series and to study frequency-domain sympathovagal behavior during ischemia. The results support the hypothesis that there are at least two distinct populations, which differ according to mechanisms and temporal patterns of ischemia.

Copyright (C) 2007 A. Smrdel and F. Jager. This is an open access article distributed under the Creative Commons Attribution License, which permits unrestricted use, distribution, and reproduction in any medium, provided the original work is properly cited.

\section{INTRODUCTION}

Ischemia is one of the most common heart diseases. It is caused by the insufficient supply of the heart muscle with the oxygen, which can cause part of the heart muscle to become electrically inactive, and can in turn lead to acute myocardial infarction, and consequently death. To further complicate the matter, up to $95 \%$ of ischemic episodes may be silent [1], while others are symptomatic. Ischemia occurs in different ischemic syndromes, such as coronary artery disease, stable angina pectoris, unstable angina, Prinzmetal's angina, transmural angina, and syndrome X [2]. The most commonly ischemia appears in patients with stable coronary artery disease. In these patients, ischemia is usually preceded with a marked increase in heart rate, and majority of episodes appear to be caused by a physical exertion, where patophysiology is connected to increased oxygen demand associated with increased myocardial contractility and blood pressure. Less common are ischemic episodes which are not preceded by an increase in heart rate. These episodes usually appear due to mental stress, where patophysiology is connected to reduced oxygen supply due to coronary vasoconstrictions. This group includes ischemic episodes of Prinzmetal's angina due to vasospasms, of unstable angina due to thrombosis, and of microvascular angina. Determining the type of ischemia (increased demand or reduced supply) for a given patient using the analysis of the long-term ambulatory electrocardiographic (ECG) records of the patient alone would not only reduce the cost, but would also represent a noninvasive alternative to current invasive techniques for optimal selection of therapy.

Previous study [3] described three different patterns of ischemic episodes in the European Society of Cardiology STT Database (ESC DB) [4]: salvo pattern, where episodes appear in short bursts; periodic pattern, where episodes appear quasiperiodically during the entire record; and sporadic pattern, where episodes appear without regularity. The hypothesis was set that different physiologic mechanisms are responsible for different patterns, namely, that the salvo patterns appear due to reduced oxygen supply caused by vasospasms, emboly, or mental stress, while the sporadic patterns appear due to increased oxygen demand caused by increased physical activity. Further study, using records of the ESC DB, tested the hypothesis [5]. The observations of this study supported the hypothesis, but the authors also pointed out that the short duration of records in the ESC DB (2 hours) does not 
permit accurate classification of records according to temporal patterns. Since then, the Long-Term ST Database (LTST DB) [6] was released. The LTST DB contains 86 24-hour expert-annotated ECG record, with a selection of records covering majority of the ischemic syndromes. Owing to the duration of records, the LTST DB enables better classification of records according to temporal patterns, and in addition, enables studies regarding diurnal changes. With numerous samples of different temporal patterns, the LTST DB allows studies of physiologic mechanisms responsible for ischemia. Preliminary study in order to test the hypothesis using the LTST DB was conducted in [7].

In this paper, we test the hypothesis, using the 24-hour records of the LTST DB, that the salvo patterns are caused due to vasospasms while the sporadic patterns appear due to physical exertion. We studied time- and frequency-domain parameters of instantaneous heart rate (IHR) before and during ischemic episodes for records with distinct temporal patterns of ischemia (salvo, periodic, sporadic) during different intervals of records (morning, day, night) and during the entire record.

\section{METHODS}

We divided each record of the LTST DB into three intervals of records (see Figure 1):

(i) night interval: time when the patient is asleep;

(ii) morning interval: a 90-minute interval, following the night interval;

(iii) day interval: the rest of the record.

Time when the patient is asleep is not provided within the LTST DB, so we identified the night interval for each record by observing trends of heart rate, which is lower and smoother during the sleep, time series of QRS complex Karhunen-Loève (KL) coefficients, in which much more sudden step changes due to axis shifts are present during the night, and noise, which is not as frequent during the sleeping period as it is during the wake period. The morning interval was defined as a 90-minute interval following the night interval. The rest of the record was considered to be the day interval. We then divided records of the LTST DB into three sets using visual examination of time series of ST segment deviation levels:

(i) the salvo-episode set which includes 6 records with distinct salvo patterns of episodes: s20021 (see Figure 2), s20151, s20171, s20291, s20301 and s20311;

(ii) the periodic-episode set which includes 12 records with periodic pattern of episodes: s20041, s20111, s20121, s20131, s20181, s20261, s20271, s20411, s20511, s20611, s30671, s30681 (see Figure 1);

(iii) the sporadic-episode set which includes 24 records with sporadic pattern of episodes: s20031, s20081, s20101 (see Figure 3), s20191, s20251, s20331, s20341, s20351, s20361, s20381, s20391, s20431, s20441, s20451, s20481, s20491, s20551, s20571, s20601, s30701, s30731, s30751, s30791, s30801.
Of the remaining 44 records, 21 showed no distinct patterns of ischemic ST segment changes (s20051, s20161, s20281, s20321, s20371, s20401, s20421, s20461, s20471, s20561, s20581, s20591, s30661, s30691, s30711, s30721, s30741, s30742, s30761, s30771 and s30781), 18 records had no significant ischemic changes (s20011, s20061, s20071, s20091, s20141, s20201, s20211, s20221, s20231, s20241, s20501, s20521, s20531, s20541, s20621, s20631, s20641 and s20651), while 5 records were excluded as duplicate records of patients already included in one of the three sets (s20272, s20273, s20274, s30732, and s30752). Multiple records belonging to the same patient exhibited the same temporal pattern, and only the first record of the same patient was included into one of the three sets.

We derived IHR time series, where abnormal beats and their neighbors were excluded. The IHR time series were then resampled ( $\Delta t=0.5 \mathrm{~s}$ ) and smoothed (3-point moving average). Next we derived time- and frequency-domain parameters of the IHR time series over several intervals before and during ischemic episodes (see Figure 4). We derived timedomain parameters by calculating the mean and standard deviation of the IHR in the intervals and two time series of the frequency-domain parameters of the IHR: fraction of the total IHR power in the low-frequency (LF) band (from $0.04 \mathrm{~Hz}$ to $0.15 \mathrm{~Hz}$ ) and fraction of the total IHR power in the highfrequency (HF) band (from $0.15 \mathrm{~Hz}$ to $0.4 \mathrm{~Hz}$ ). Using these time series, we computed mean and standard deviation of the frequency-domain parameters in intervals before and during ischemia.

To derive the frequency-domain parameters of the IHR time series we used the adaptive autoregressive method with recursive least-square (RLS) algorithm $[8,9]$. The advantage of this method is that it is able to adapt to nonstationary behavior of time series being analyzed by adaptively estimating autoregressive (AR) coefficients for each sample. The AR coefficients are updated on the basis of previous ones and a forgetting factor $\lambda$. Changes of the signal spectra characteristics are tracked by weighting the performance index $\epsilon$ of the RLS-based predictor:

$$
\epsilon=\sum_{i=0}^{n} \lambda^{n-i} e(i)^{2}
$$

where $0<\lambda \leq 1$ is the forgetting factor, $n$ is the index of the last sample considered, and $e(i)$ is the estimation error,

$$
e(i)=y(i)-\hat{y}(i) \text {. }
$$

The predicted signal $\hat{y}(i)$ can be estimated as

$$
\hat{y}(i)=\sum_{m=1}^{M} a_{m}(n) y(i-m)=(\mathbf{a}(n))^{T} \mathbf{y}(i-1),
$$

where $\mathbf{a}(n)$ are the prediction coefficients, $M$ is the order of the model, and

$$
\mathbf{y}(i-1)=\left[\begin{array}{c}
y(i-1) \\
y(i-2) \\
\vdots \\
y(i-M)
\end{array}\right]
$$




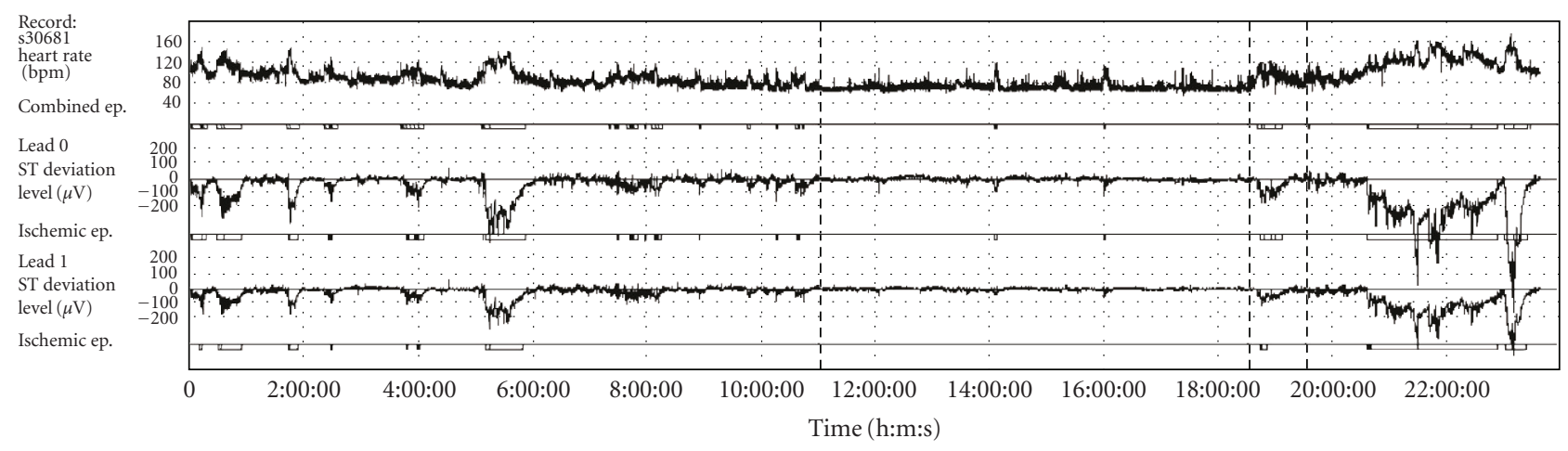

FIGURE 1: Time trends of a three-lead record s30681 (first two leads shown) from the periodic-episode set (all 24 hours of the recording are shown, recording starts at 11:35:00, which corresponds to the time 0:00 in this figure). Vertical lines separate day, night, morning, and day intervals. From top to bottom: heart rate (bpm); ischemic episodes from all leads, combined in the sense of logical OR function; ST segment deviation for lead $0(\mu \mathrm{V})$; ischemic episodes for lead 0 ; ST segment deviation for lead $1(\mu \mathrm{V})$; ischemic episodes for lead 1.

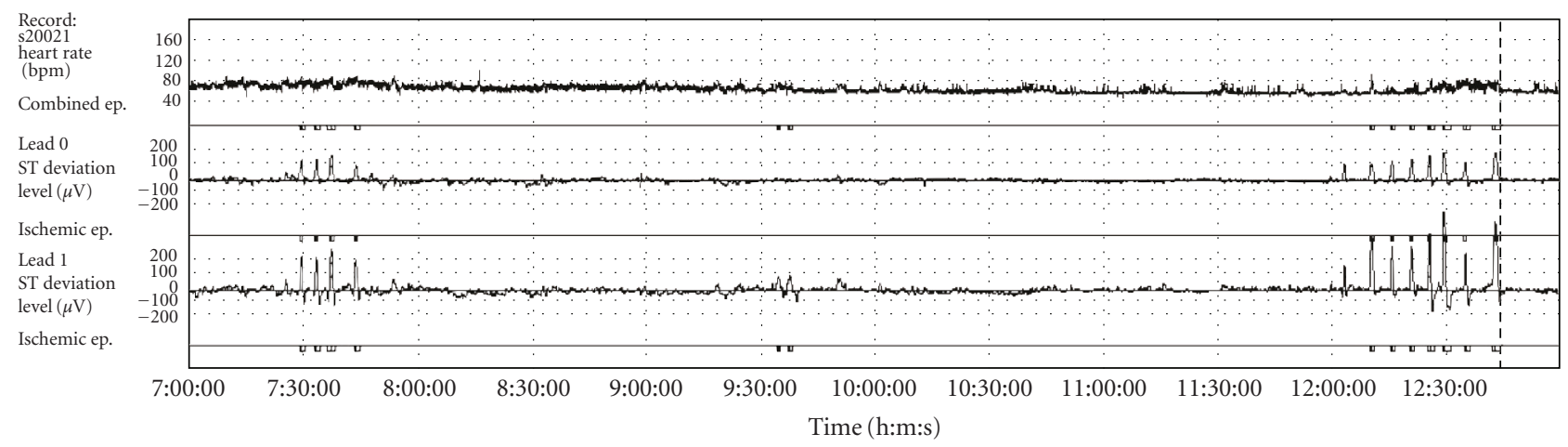

FIgURE 2: Time trends of a two-lead record s20021 from the salvo-episode set (6-hour excerpt from 24-hour record is shown, recording starts at 11:00:00, corresponding to the time 0:00 at the beginning of this record, the last few hours of the day interval and the beginning of the night interval are shown, which are separated by a vertical line). (For the legends, see caption of Figure 1.)

The performance index must be minimized according to the adaptive prediction coefficients vector $(\delta \epsilon / \delta \mathbf{a}=0)$, thus satisfying the following expression:

$$
\mathbf{a}(n)=(\mathbf{R}(n))^{-1} \mathbf{p}(n),
$$

where $\mathbf{R}(n)$ represents the autocorrelation matrix of $\{y(i)\}$ signal calculated on $M$ samples over a window of $n$ samples:

$$
\mathbf{R}(n)=\sum_{i=0}^{n} \lambda^{n-i} \mathbf{y}(i-1)(\mathbf{y}(i-1))^{T},
$$

and

$$
\mathbf{p}(n)=\sum_{i=0}^{n} \lambda^{n-i} y(i) \mathbf{y}(i-1)
$$

From this equation, the first vector of prediction coefficients, $\mathbf{a}(n)$, is calculated. All other vectors are calculated, without minimizing the performance index, following the recursive formula:

$$
\mathbf{a}(n)=\mathbf{a}(n-1)-e\left(\frac{n}{n-1}\right) \mathbf{g}(n)
$$

where

$$
e\left(\frac{n}{n-1}\right)=y(n)-(\mathbf{a}(n-1))^{T} \mathbf{y}(n-1)
$$

represents the estimation error and $\mathbf{g}(n)$ represents the Kalman gain vector,

$$
\mathbf{g}(n)=\frac{\mathbf{Q}(n-1) \mathbf{y}(n-1)}{\lambda+(\mathbf{y}(n-1))^{T} \mathbf{Q}(n-1) \mathbf{y}(n-1)},
$$

and $\mathbf{Q}(n)=\mathbf{R}(n)^{-1}$. For each sample, the $\mathbf{Q}(n)$ is recursively updated,

$$
\mathbf{Q}(n)=\frac{\left(\mathbf{I}-\mathbf{g}(n)(\mathbf{y}(n-1))^{T}\right) \mathbf{Q}(n-1)}{\lambda},
$$

where I is the unit matrix. The power spectral density $S(\omega, n)$ can be estimated at the $n$th sample from the AR coefficients $\mathbf{a}(n)$ :

$$
S(\omega, n)=\frac{\sigma_{e}^{2}(n)}{\left|1+\sum_{i=1}^{M} a_{i}(n) e^{-j i \omega \Delta t}\right|^{2}},
$$




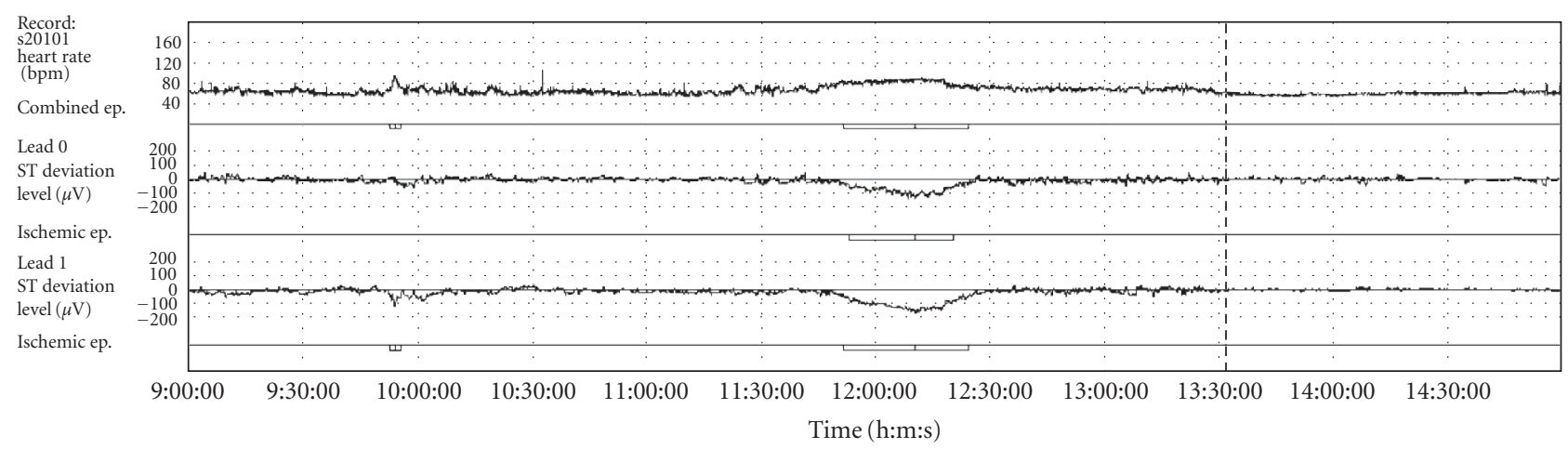

Figure 3: Time trends of a two-lead record s20101 from the sporadic-episode set (6-hour excerpt from the 24-hour record is shown, recording starts at 9:33:00, corresponding to the time 0:00 at the beginning of this record, the last few hours of the day interval and the beginning of the night interval are shown, which are separated by a vertical line). (For the legends, see caption of Figure 1.)

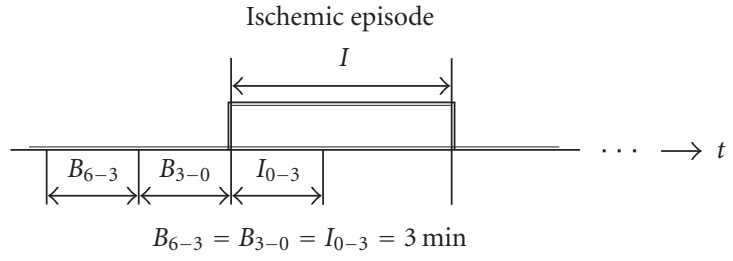

Figure 4: Time intervals before and during ischemic episodes, used for the time- and frequency-domain analyses.

where $\sigma_{e}^{2}(n)$ is the noise power at the $n$th sample and $\Delta t$ is the sampling interval. A previous study [8] found that a model order, $M$, of 12 allows acceptable discrimination of the frequencies of interest. With higher order, the model is more adapted and also more prone to noise, while computation is slower. The number of samples, participating in the power spectra estimation, depends on the forgetting factor $\lambda$. For $\lambda=1$, the entire time series contributes to the power spectra estimation, which is acceptable for the stationary time series, while for the nonstationary time series values of $\lambda$ smaller than 1 are required, which permit faster adaption. With low values of $\lambda$, the system better adapts to changes but is also more sensitive to noise, which demands value of $\lambda$ to be close to 1 . This allows for longer windows, thus making the system more insensitive to noise but also less able to track rapid changes. The border frequencies of LF and HF bands $(0.04-0.15 \mathrm{~Hz}$ and $0.15-0.4 \mathrm{~Hz}$, resp.) correspond to periods of 25 seconds $(0.04 \mathrm{~Hz}), 6.7$ seconds $(0.15 \mathrm{~Hz})$, and 2.5 seconds $(0.4 \mathrm{~Hz})$. We chose the values of $\lambda=0.985$, which corresponds to exponentially weighted window of 33 seconds $(\lambda /(1-\lambda))$. This window length also corresponds to the shortest annotated episode in the LTST $\mathrm{DB}$, which is 30 seconds in duration. The fraction of the total IHR power in the LF band was used to estimate the sympathetic activity, while the fraction of the total IHR power in the HF band was used to estimate the vagal activity.

From obtained time- and frequency-domain measurements, we derived aggregate average statistics for the sets dur- ing all intervals of records. To assess significant differences in intervals before and during ischemia, we used statistical method one-way ANalysis Of VAriance (ANOVA) [10], while to assess significant differences between pairs of sets during intervals of records prior to and during ischemia, we used Student $t$ test [11], which is a special case of one-way ANOVA for two sets. A value of $P<.01$ was considered significant.

\section{RESULTS}

A number of ischemic episodes and percentages of ischemia duration per set for different intervals of records are shown in Table 1. The incidence of ischemia as compared to other intervals of records is the greatest for all sets during the morning interval, while the lowest incidence is during the night interval. For the salvo-episode set the changes in incidence throughout different intervals of records are minor. During the morning interval, the ischemia duration was only slightly higher than during the day and night intervals. For the periodic- and sporadic-episode sets, the changes during different intervals are much more prominent. For these two sets, the percentage of ischemia duration during the morning interval is almost twice as high as it is during other intervals of records. For the sporadic-episode set, only two episodes appear during the night interval, when there is no physical activity.

Table 2 shows aggregate average heart rates for all sets during the intervals of records. The lowest heart rate is exhibited for the salvo-episode set. Generally, the highest heart rate for each set is during the morning interval, except for the periodic-episode set, when slightly higher heart rate is during the day interval. For the periodic- and sporadic-episode set the heart rate during the night interval significantly differs from that during the morning ( $t$ test: $P<.005$ and $P<.001$, resp.) and day interval ( $t$ test: $P<.005$ and $P<.001$, resp.), indicating much lower heart rate during the night interval as it is during the morning and day intervals. Table 3 shows aggregate average ratios of the mean heart rate of the intervals $I_{0-3}$ and $B_{3-0}$. The lowest ratio of the mean heart rate is during the day interval for all the sets, while the highest is during 
TABLE 1: Number of ischemic episodes and percentages of ischemia duration (bracketed) per set for different intervals of records.

\begin{tabular}{l|l|l|c|c}
\hline Number of episodes & Morning & Day & Night & Entire record \\
\hline Salvo-episode set & $18(9 \%)$ & $104(6 \%)$ & $60(6 \%)$ & $182(6 \%)$ \\
Periodic-episode set & $27(34 \%)$ & $195(17 \%)$ & $60(8 \%)$ & $282(15 \%)$ \\
Sporadic-episode set & $23(13 \%)$ & $136(8 \%)$ & $2(0 \%)$ & $161(6 \%)$ \\
\hline
\end{tabular}

TABLE 2: Aggregate average heart rates for all sets during the intervals of records. Standard deviations are bracketed.

\begin{tabular}{l|lllc}
\hline Heart rate $(\mathrm{bpm})$ & \multicolumn{1}{|c}{ Morning } & \multicolumn{1}{c}{ Day } & \multicolumn{1}{c}{ Night } & Entire record \\
\hline Salvo-episode set & $74.3(13)$ & $69.7(10)$ & $59.1(10)$ & $66.2(11)$ \\
Periodic-episode set & $78.2^{\ddagger_{1}}(11)$ & $79.1^{\ddagger_{2}}(11)$ & $66.0(7)$ & $74.7(9)$ \\
Sporadic-episode set & $79.3^{\star_{1}}(11)$ & $76.9^{\star_{2}}(8)$ & $62.7^{\star_{3}}(7)$ & $72.4(7)$ \\
\hline
\end{tabular}

${ }^{{ }_{1}} P<.005$ (morning, night, periodic). ${ }^{\ddagger_{2}} P<.005$ (day, night, periodic). ${ }^{{ }_{1}} P<.001$ (morning, night, sporadic). ${ }^{\star} 2 P<.001$ (day, night, sporadic). ${ }^{{ }_{3}} P<.001$ (night, entire record, sporadic).

TABle 3: Aggregate average ratios of the mean heart rate of the intervals $I_{0-3}\left(\mathrm{HR}_{I_{0-3}}\right)$ and $B_{3-0}\left(\mathrm{HR}_{B_{3-0}}\right)$.

\begin{tabular}{l|cccc}
\hline $\mathrm{HR}_{I_{0-3}} / \mathrm{HR}_{B_{3-0}}$ & Morning & Day & Night & Entire record \\
\hline Salvo-episode set & 1.054 & 1.041 & 1.048 & 1.048 \\
Periodic-episode set & 1.156 & 1.096 & 1.178 & 1.139 \\
Sporadic-episode set & 1.122 & 1.117 & 1.264 & 1.126 \\
\hline
\end{tabular}

the morning interval for the salvo-episode set and during the night interval for the periodic- and sporadic-episode sets.

Figure 5 shows aggregate average heart rates for the sets during intervals of records and for the entire record before and during ischemia. Generally, the lowest heart rate is exhibited for the salvo-episode set throughout the intervals of records and for the entire record for intervals before and during ischemia, while the highest heart rate is exhibited for the sporadic-episode set. In the intervals of records and for the entire record, for all the sets, the heart rate in the intervals $B_{3-0}$ and $I_{0-3}$ rises but this increase is the least prominent for the salvo-episode set. During the morning interval before the ischemia onset, the heart rate for the salvo- and periodicepisode set is approximately equal, while during ischemia the heart rate for the periodic-episode set is much higher as it is for the salvo-episode set. In the day interval, during the intervals $B_{3-0}$ and $I_{0-3}$, the heart rate for the salvo-episode set significantly differs from that of the sporadic-episode set $(t$ test: $P<.005$ in both cases), indicating that different mechanisms might be responsible for ischemia in these two sets. In the night interval, the heart rate for the salvo-episode set is much lower than the heart rate for the periodic- and sporadic-episode sets, but for all three sets, and not just for the salvo-episode set, the heart rate starts to rise only during ischemia. The overall results (entire record) show that the heart rate for the salvo-episode set starts to rise after the ischemia onset, while for the periodic- and sporadic-episode sets the heart rate starts to rise before ischemia onset. For the entire record, during the intervals $B_{3-0}$ and $I_{0-3}$, the heart rate for the salvo-episode set significantly differs from that of the sporadic-episode set ( $t$ test: $P<.001$ in both cases). During the day interval and for the entire record, the heart rate for the sporadic-episode set significantly changes over intervals prior to and during ischemia (one-way ANOVA: $P<.001$ in both cases). The significant differences in average heart rates for salvo- and sporadic-episode sets indicate that the ischemia in both groups might be triggered by different mechanisms. In addition, the significant change in heart rate before and during ischemia for sporadic-episode set indicates that ischemia in this set could be caused by physical exertion.

Figure 6 shows aggregate average normalized LF powers for the sets during intervals of records and for the entire record before and during ischemia. Generally, the highest LF power during intervals of records and for the entire record is exhibited for the salvo-episode set, while the lowest LF power is exhibited for the sporadic-episode set. For all the sets during intervals of records and for the entire record, the LF power drops at the ischemia onset as compared to the interval prior to ischemia, except for the salvo-episode set during the night interval. The greatest changes of the LF power appear during the morning interval for all the sets, and also during the night interval for the sporadic-episode set. (Note that there are only two episodes present in this set during the night interval.) In the day interval and for the entire record, the LF power for the salvo-episode set significantly differs from that of the sporadic-episode set during the intervals $B_{3-0}$ ( $t$ test: $P<.001$ in both cases) and $I_{0-3}$ ( $t$ test: $P<.001$ in both cases), indicating different sympathetic activity in both sets, which might be explained with different mechanisms. During the day interval and for the entire record, the LF power for the sporadic-episode set significantly changes over intervals prior to and during ischemia (one-way ANOVA: $P<.001$ in both cases).

Figure 7 shows aggregate average normalized HF powers for the sets during intervals of records and for the entire record, before and during ischemia. During the intervals of records and for the entire record, the salvoepisode set exhibits generally the highest HF power, while 


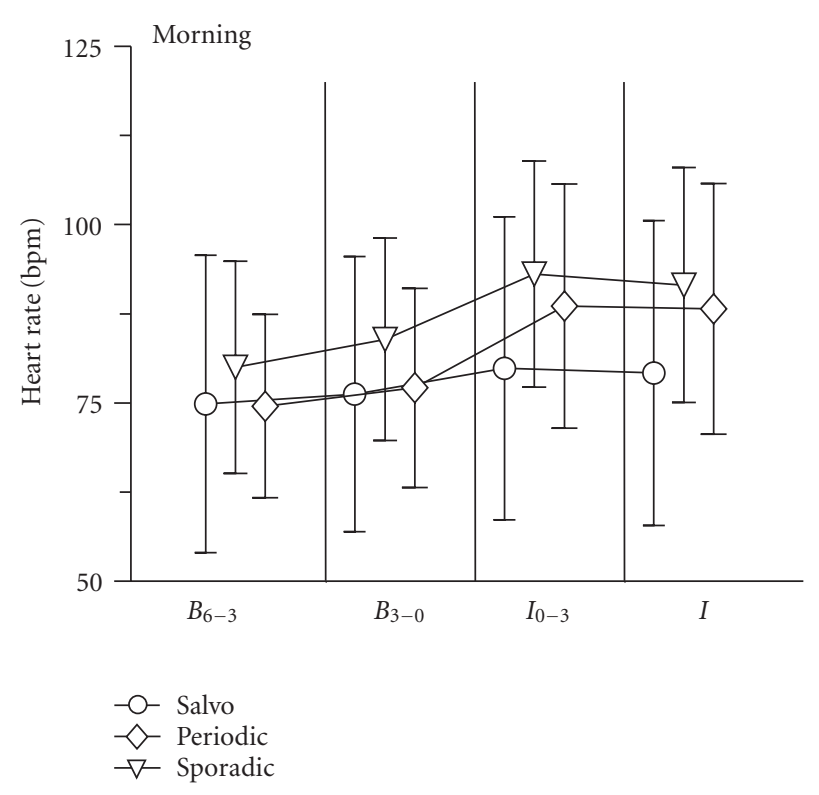

(a)

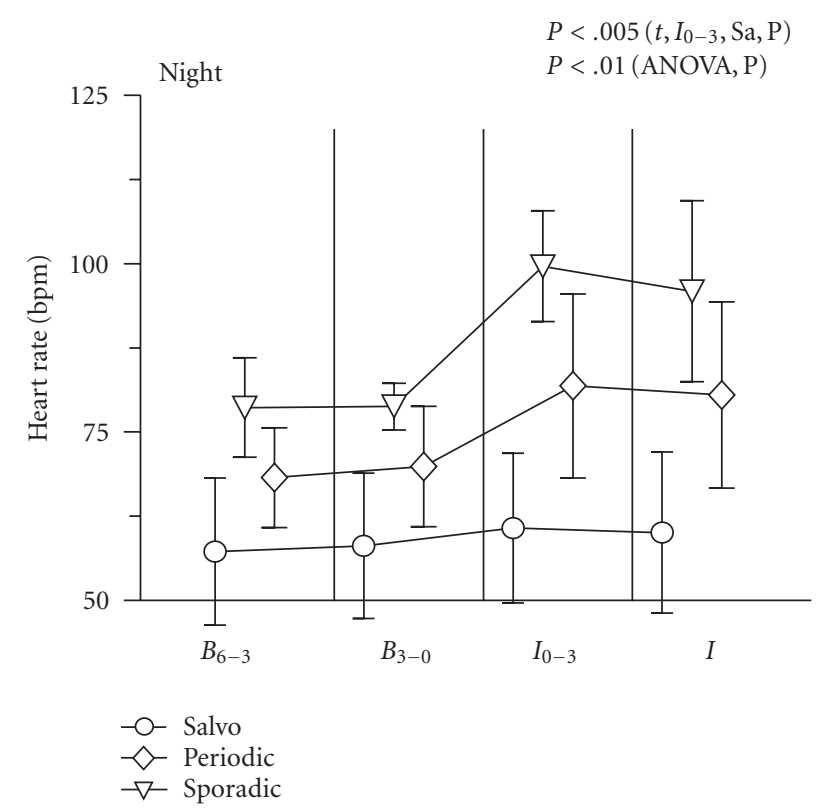

(c)

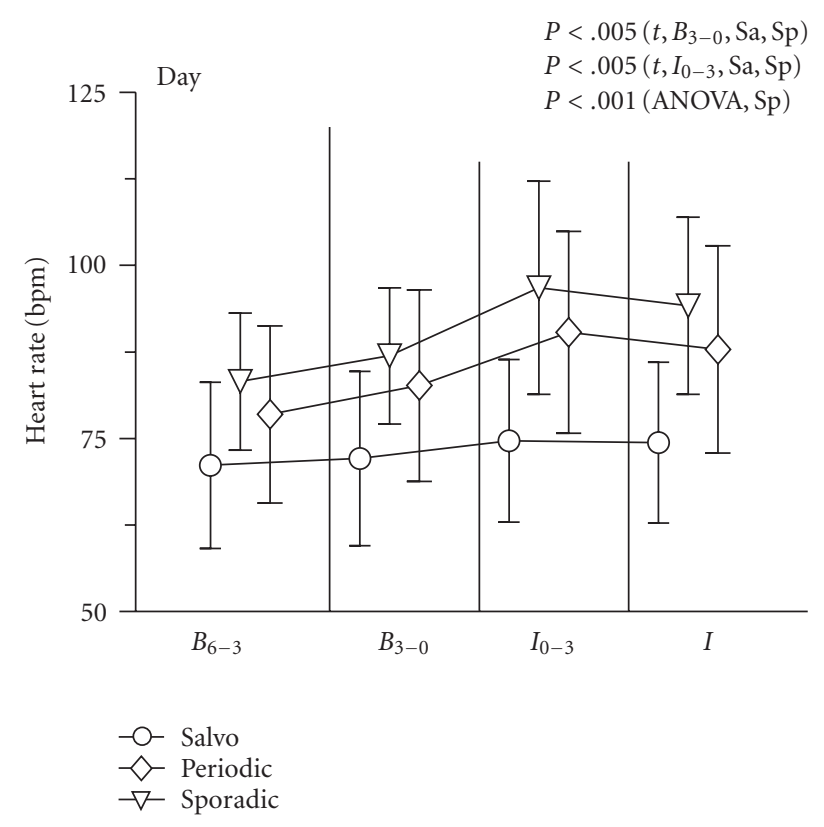

(b)

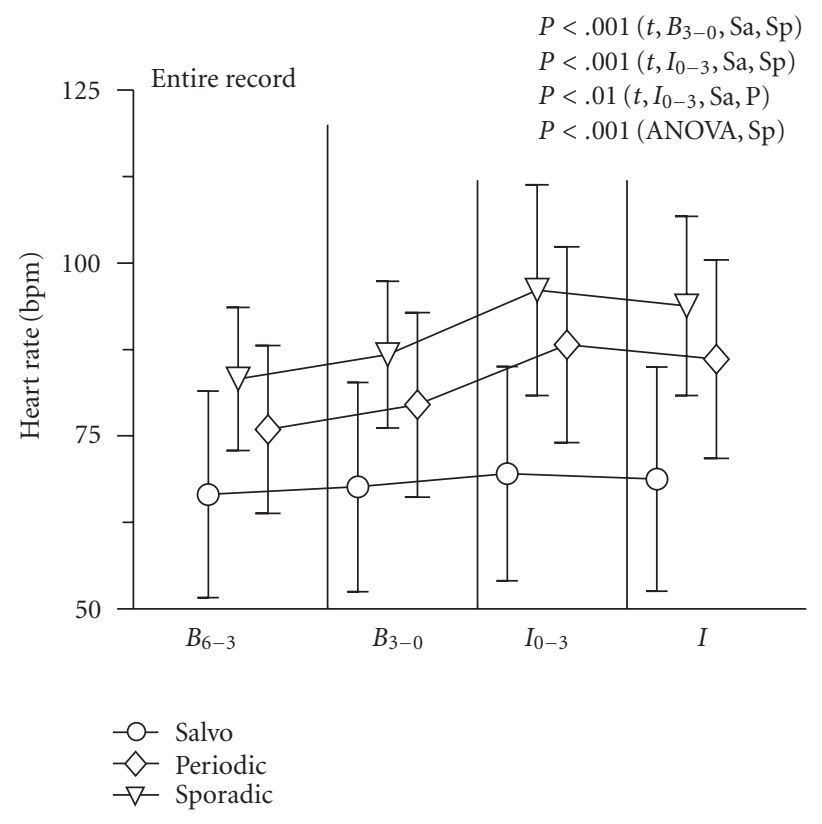

(d)

FIGURE 5: Aggregate average heart rates for the sets during intervals of records and for the entire record before and during ischemia. Vertical bars indicate aggregate average standard deviations within the intervals $B_{6-3}$ (from 6 minutes to 3 minutes prior to ischemia), $B_{3-0}$ (from 3 minutes prior to ischemia to ischemia onset), $I_{0-3}$ (first 3 minutes of ischemia), and $I$ (during ischemia). Sa indicates salvo-episode set, $\mathrm{P}$ indicates periodic-episode set, $\mathrm{Sp}$ indicates sporadic-episode set. $t$ indicates Student $t$ test over indicated interval for indicated sets. ANOVA indicates one-way ANOVA for the indicated group over intervals $B_{6-3}, B_{3-0}, I_{0-3}$, and $I$.

the sporadic-episode set exhibits generally the lowest HF power. During the morning and day interval, and for the entire record, the HF power for all sets slightly drops at the beginning of ischemia as compared to the interval prior to ischemia onset, except for the salvo-episode set during the morning interval, when HF power remains unchanged. During the night interval, the HF power for the salvo- and periodic-episode sets slightly rises at the beginning of ischemia as compared to the interval prior to ischemia onset, while for the sporadic-episode set the HF power drops. During the day interval and for the entire record, the HF power for the salvo-episode set significantly differs from that of the sporadic-episode set during the intervals $B_{3-0}(t$ test: $P<.001$ and $P<.01$, resp. $)$ and $I_{0-3}(t$ test: $P<.001$ in 


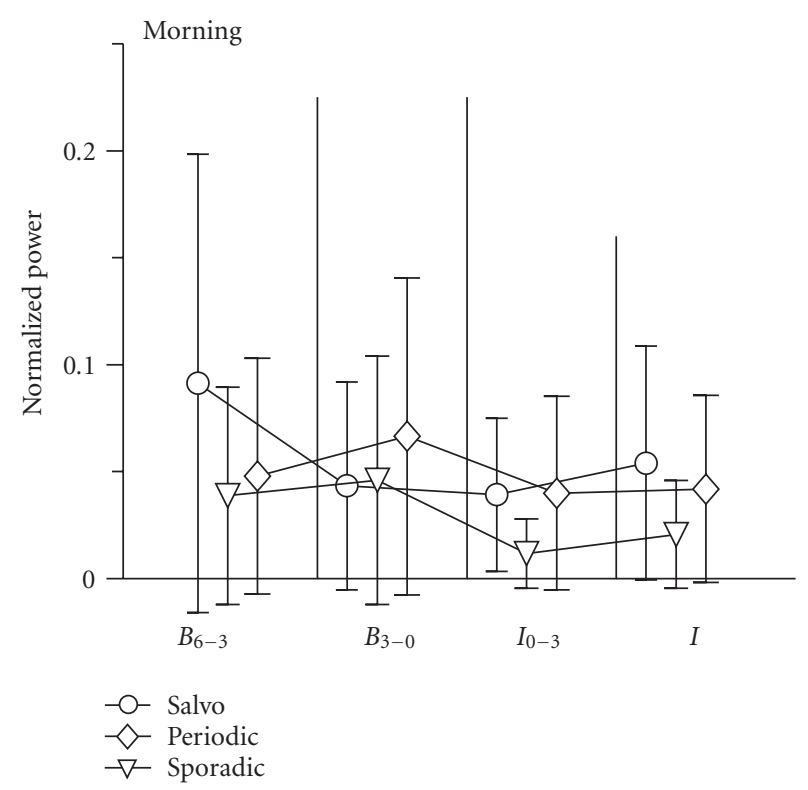

(a)

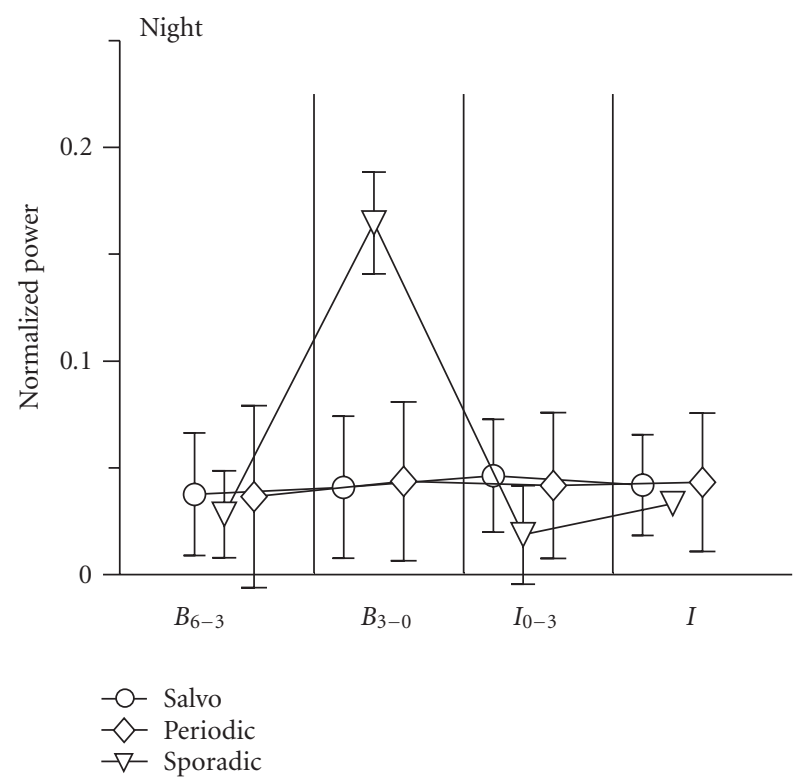

(c)

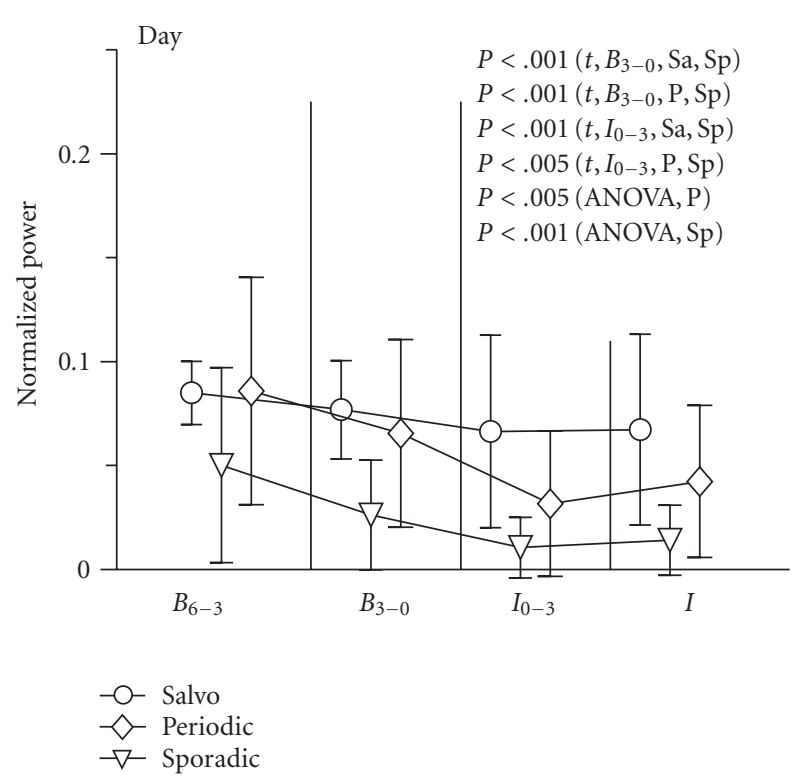

(b)

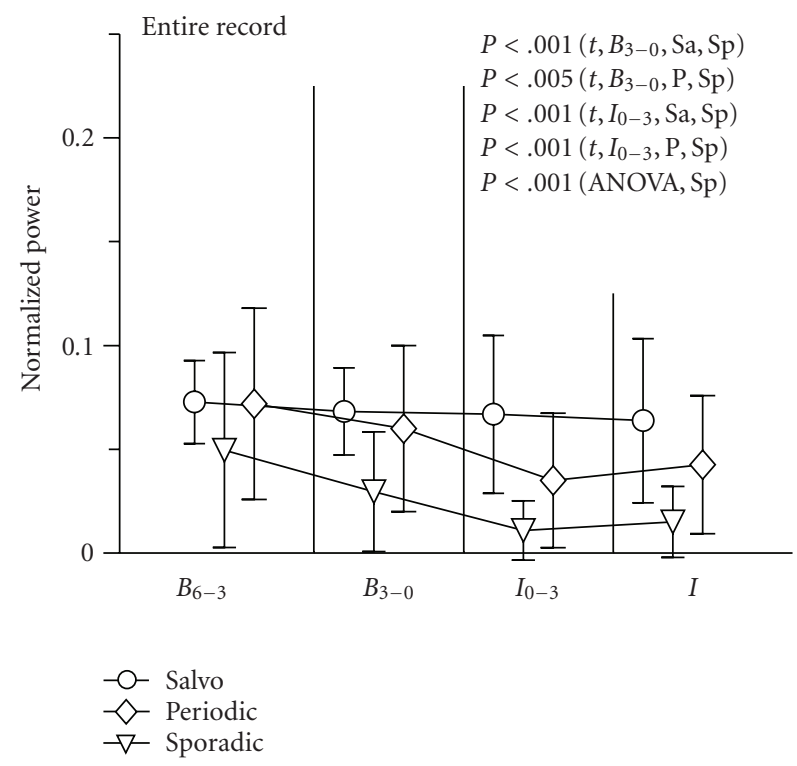

(d)

FIgURE 6: Aggregate average normalized LF powers for the sets during intervals of records and for the entire record before and during ischemia. Vertical bars indicate aggregate average standard deviations within the intervals prior to and during ischemia. (For the legends, see caption of Figure 5.)

both cases), indicating different vagal activities in both sets, which could be explained by different mechanisms involved in triggering the ischemia. During the day interval and for the entire record, the HF power for the sporadic-episode set significantly changes over intervals prior to and during ischemia (one-way ANOVA: $P<.001$ in both cases). These results, together with the results for the LF power for sporadicepisode set, indicate that ischemia in sporadic-episode set would seem to be characterized by a high degree of variability, which is consistent with observations in [12], while is- chemia in salvo-episode set does not exhibit such degree of variability.

\section{DISCUSSION}

The time- and frequency-domain results, obtained using 24hour annotated records of the LTST DB, support our hypothesis, that the sporadic ischemic episodes appear due to physical exertion, while the salvos of ischemic episodes are result of coronary vasoconstrictions and vasospasms. 


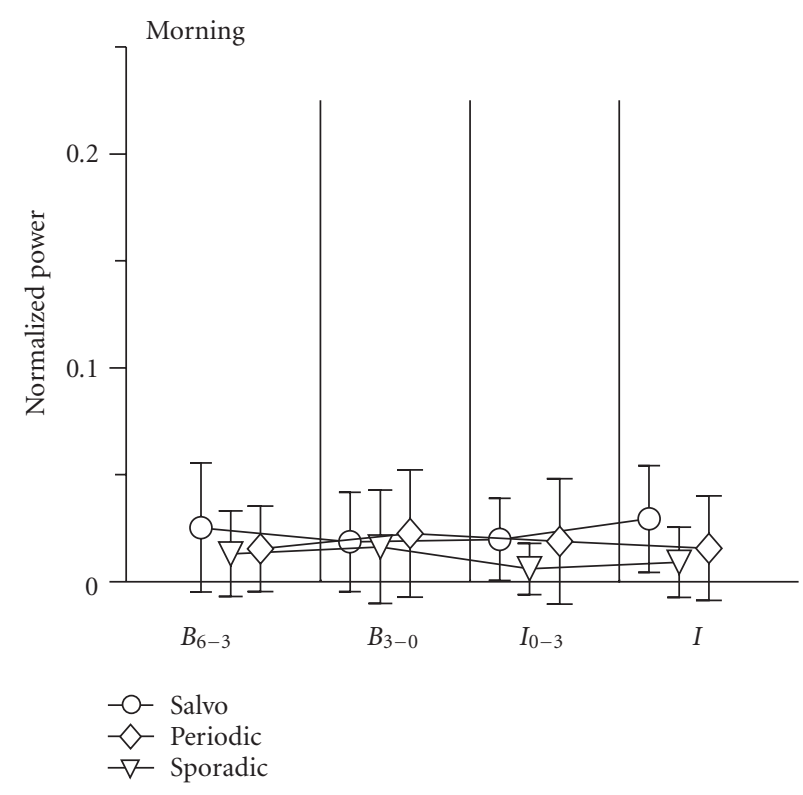

(a)

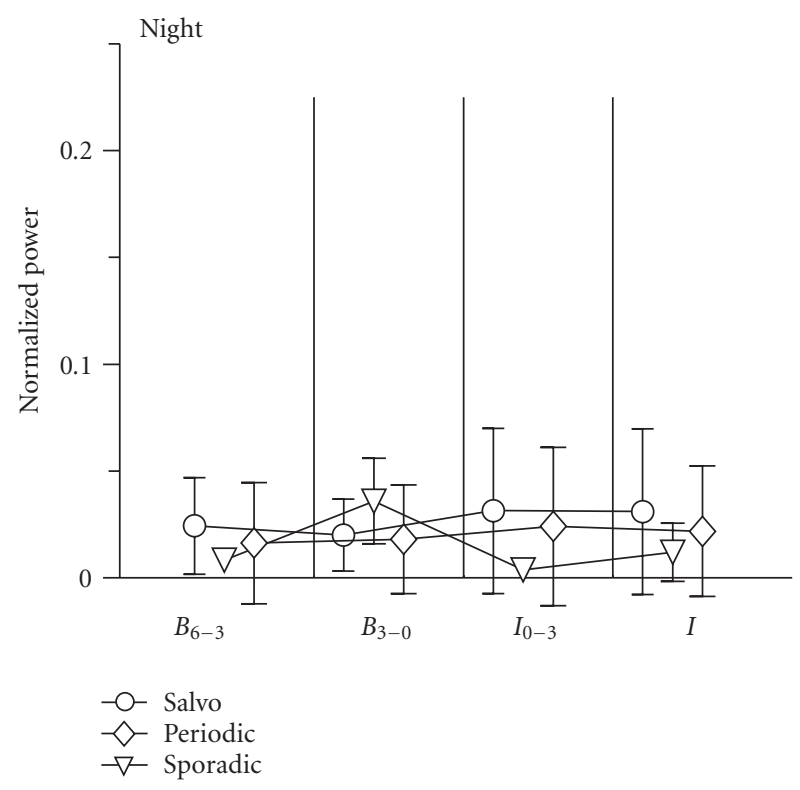

(c)

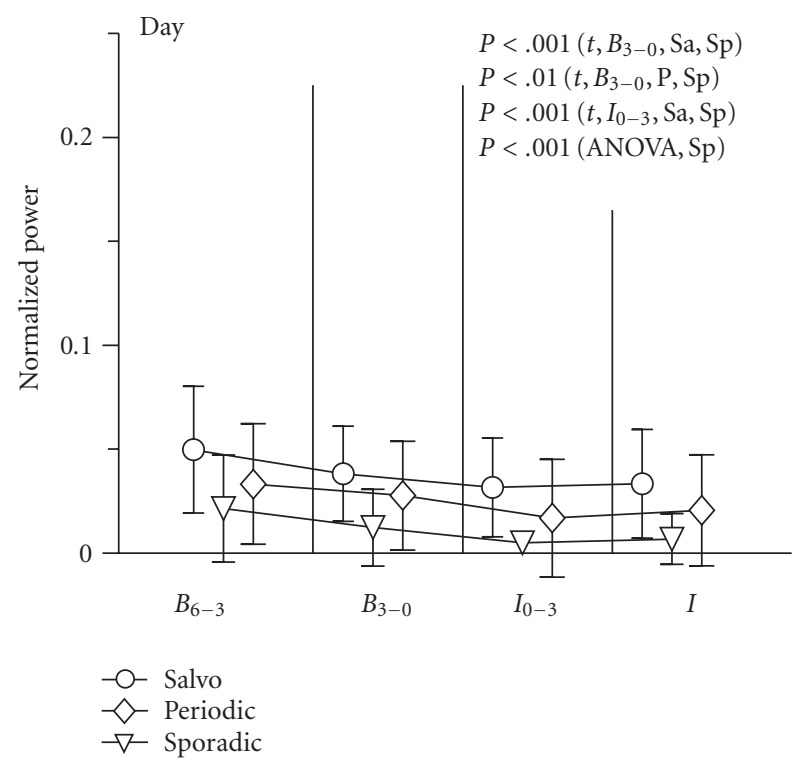

(b)

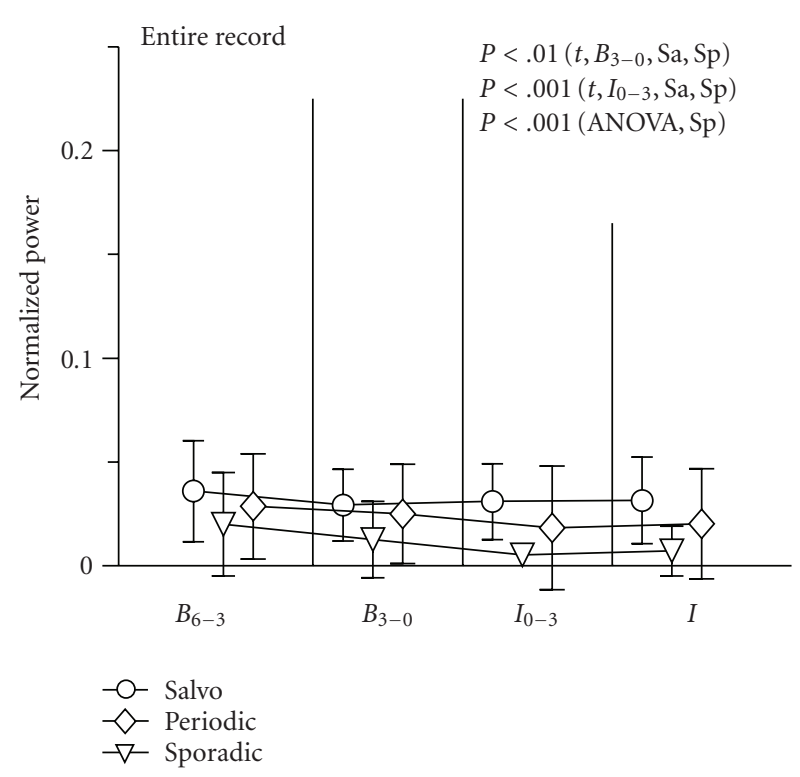

(d)

FIgURE 7: Aggregate average normalized HF powers for the sets during intervals of records and for the entire record before and during ischemia. Vertical bars indicate aggregate average standard deviations within the intervals prior to and during ischemia. (For the legends, see caption of Figure 5.)

Results show that the records in the salvo-episode set have generally lower heart rate and standard deviation compared to the records in the sporadic-episode set. We also observed a notable increase of the heart rate prior to ischemia onset for the sporadic-episode set which indicates that the heart rate starts to increase in response to physical exertion in order to satisfy increased oxygen demand, and only after that ischemia occurs. For the salvo-episode set, we observed only slight increase in the heart rate before ischemia onset indicating that ischemia in this set is not a result of increased physical exertion (increase in oxygen demand), but is rather a result of insufficient oxygen supply, which might occur due to vasospasms and vasoconstrictions. For the sporadic-episode set, the heart rate statistically significantly rises at the beginning of an ischemic episode compared to the interval prior to ischemia onset (in the day interval and for the entire record), 
while the increase in the heart rate for the salvo-episode set is not significant, also indicating that ischemia in this set is not a result of physical exertion. Slight increase in heart rate for the salvo-episode set might be interpreted as an attempt to increase the blood supply to a heart muscle by an increase in heart rate. Interestingly, the records in the periodic-episode set exhibit similar changes of the heart rate before and during ischemia as the sporadic-episode set, but the heart rate in these intervals is much lower (the heart rate for the periodicepisode set is approximately from 3.5 to 18 beats per minute lower than it is for the sporadic-episode set), while the heart rate during the day and night intervals and during the entire record is higher than it is for the sporadic-episode set. The time-domain results also show that in all sets, the incidence of ischemia is the largest during the morning interval, and the lowest during the night interval, which is in agreement with observations in $[13,14]$. This indicates a much greater risk of ischemia in the morning interval than during other intervals of day, and might be attributed to the lower ischemia threshold during the morning interval [15].

The frequency-domain results show change of the LF power (sympathetic activity) for the sporadic-episode set before the ischemia onset and significant change of the LF power at the beginning of ischemia compared to the interval immediately prior to ischemia onset (except during the night interval when only two episodes appeared in the sporadicepisode set). For the HF power (vagal activity), we observed smaller changes in intervals before and during ischemia especially for the salvo-episode set, while for the sporadic-episode set we observed significant decrease in activity at the beginning of ischemia as compared to the interval immediately prior to ischemia onset during the day interval and for the entire record. The results regarding sympathetic and vagal activities in our study seem in agreement with several previous studies $[14,16,17]$, which also described marked changes in sympathetic and/or vagal activity during the ischemia.

Examination of the prior clinical information for the patients showed that all the patients, whose records were inserted in the salvo-episode set, had a Prinzmetal's angina (four of them had also a coronary artery disease). The patients, whose records were inserted in the periodic-episode set, had mostly coronary artery disease, usually in combination with other diseases. The majority of the patients, whose records were inserted in the sporadic-episode set, had a coronary artery disease (22 of 24, one of those 22 had also a Prinzmetal's angina), one patient in this set suffered from syndrome X and one had palpitations. Regarding this, we may conclude that the salvo patterns usually appear in patients with severe cases of heart disease, such as Prinzmetal's angina, while sporadic patterns appear in patients with milder forms of heart disease, such as coronary artery disease.

The patophysiology of ischemia triggered by vasospasms or mental stress is difficult to describe. One possible explanation is that during the postexcitation phase, the blood pressure may drop abruptly and recovery of heart rate and contractility may be delayed. We may further speculate that pe- riodic platelet adhesion and breakoff may account for recurrent vasospasms. Also a recent study suggests that vagal withdrawal precedes the onset of mental stress-induced ischemia [18]. On the other hand, the patophysiology of the ischemia triggered by the exertion is easier to describe. Usually, the ischemia is preceded by an increase in heart rate due to physical exertion and an increase (morning and night intervals) in sympathetic activity. In this settings, we would expect increased contractility, blood pressure, metabolic demand, and ischemia, instead of local coronary vasodilation and increased coronary flow. In addition, ischemia also activates complex pressor and depressor reflexes, which may alter sympathetic and vagal inputs to the cardiovascular control system. The sympathovagal behavior in the sporadic-episode set is consistent with the patophysiology of effort angina.

One of the limitations of this study is the determination of the time, when patient was asleep. For the reliability of the correct determination of the night interval, we would require the diary of activities. Since this information is not available within the LTST DB, we had to rely on empirical knowledge of heart rate changes, body position changes, and noise in signal. We also used the data about time when the recording was started, which is available in the LTST DB, to verify that the time, when patient was asleep, was plausible. Also, our results regarding changes of heart rate during intervals of records show significantly lower heart rates during the night interval as compared to day and morning intervals, suggesting that the night interval (time when patient was asleep) was determined correctly. Nevertheless, the existence of the diary of activities would be beneficial in validating our technique for determination of night interval. The other limitation is a small number of records. Although our study included great number of ischemic episodes (625), not all intervals of the records were well represented. During the night interval, there were only two ischemic episodes for sporadicepisode set, which does not enable statistical interpretation. To further strengthen our findings, more records with distinct temporal patterns should be included in the future, although the sporadic-episode set probably would not contain much more records during the night interval when there is no physical activity.

\section{CONCLUSIONS}

Using the 24-hour records from the LTST DB, we tested the hypothesis that different physiologic mechanisms are responsible for different temporal patterns of ischemic episodes. The obtained results lead us to conclude that different mechanisms are responsible for salvo and for sporadic patterns of ischemic episodes, namely, that physical exertion causes sporadic patterns, while vasospasms and vasoconstrictions cause salvo patterns.

In this study, we assumed that only neurogenic factors affect coronary regulation. In acute spastic angina, local endothelial factors also regulate and modify blood flow [19]. This additional factors may result in modified response. Due to those factors, it may be inappropriate to describe the patophysiology of the salvo-episode set only in terms of sympathovagal influence. 


\section{REFERENCES}

[1] T. C. Andrews and P. H. Stone, "Recent developments in the understanding and management of angina pectoris in patients with stable coronary artery disease," Current Opinion in Cardiology, vol. 6, no. 4, pp. 503-510, 1991.

[2] A. A. Quyyumi, "Current concepts of pathophysiology, circadian patterns, and vasoreactive factors associated with myocardial ischemia detected by ambulatory electrocardiography," Cardiology Clinics, vol. 10, no. 3, pp. 403-415, 1992.

[3] F. Jager, G. B. Moody, and R. G. Mark, "Characterization of transient ischemic and non-ischemic ST segment changes," in Proceedings of 22th Annual Meeting on Computers in Cardiology, pp. 721-724, Vienna, Austria, September 1995.

[4] A. Taddei, G. Distante, M. Emdin, et al., "The European STT database: standard for evaluating systems for the analysis of ST-T changes in ambulatory electrocardiography," European Heart Journal, vol. 13, no. 9, pp. 1164-1172, 1992.

[5] F. Jager, G. B. Moody, G. Antolič, D. Mašič, and R. G. Mark, "Sympatho-vagal correlates of transient ischemia in ambulatory patients," in Proceedings of 24th Annual Meeting on Computers in Cardiology, pp. 387-390, Lund, Sweden, September 1997.

[6] F. Jager, A. Taddei, G. B. Moody, et al., "Long-term ST database: a reference for the development and evaluation of automated ischaemia detectors and for the study of the dynamics of myocardial ischaemia," Medical and Biological Engineering and Computing, vol. 41, no. 2, pp. 172-182, 2003.

[7] A. Smrdel and F. Jager, "Diurnal changes of heart rate and sympatho-vagal activity for temporal patterns of transient ischemia," in Proceedings of 32th Annual Meeting on Computers in Cardiology, pp. 857-860, Lyon, France, September 2005.

[8] A. M. Bianchi, L. Mainardi, E. Petrucci, M. G. Signorini, M. Mainardi, and S. Cerutti, "Time-variant power spectrum analysis for the detection of transient episodes in HRV signal," IEEE Transactions on Biomedical Engineering, vol. 40, no. 2, pp. 136-144, 1993.

[9] M. Akay, Biomedical Signal Processing, Academic Press, San Diego, Calif, USA, 1994.

[10] A. G. Bluman, Elementary Statistics: A Brief Version, MacGrawHill, New York, NY, USA, 3rd edition, 2006.

[11] W. H. Press, S. A. Teukolsky, W. T. Vetterling, and B. P. Flannery, Numerical Recipes in $\mathrm{C}++$, the Art of Scientific Computing, Cambridge University Press, Cambridge, Mass, USA, 2nd edition, 2002.

[12] L. G. Gamero, J. Vila, and F. Palacios, "Wavelet transform analysis of heart rate variability during mycardial ischaemia," Medical and Biological Engineering and Computing, vol. 40, no. 1, pp. 72-78, 2002.

[13] J. D. Parker, M. A. Testa, A. H. Jimenez, et al., "Morning increase in ambulatory ischemia in patients with stable coronary artery disease: importance of physical activity and increased cardiac demand," Circulation, vol. 89, no. 2, pp. 604-614, 1994.

[14] A. J. van Boven, J. Brouwer, H. J. G. M. Crijns, J. Haaksma, and K. I. Lie, "Differential autonomic mechanisms underlying early morning and daytime transient myocardial ischaemia in patients with stable coronary artery disease," British Heart Journal, vol. 73, no. 2, pp. 134-138, 1995.

[15] A. A. Quyyumi, J. A. Panza, J. G. Diodati, E. Lakatos, and S. E. Epstein, "Circadian variation in ischemic threshold: a mechanism underlying the circadian variation in ischemic events," Circulation, vol. 86, no. 1, pp. 22-28, 1992.

[16] Y. Goseki, T. Matsubara, N. Takahashi, T. Takeuchi, and C. Ibukiyama, "Heart rate variability before the occurrence of silent myocardial ischemia during ambulatory monitoring," American Journal of Cardiology, vol. 73, no. 12, pp. 845-849, 1994.

[17] S. Cerutti, A. M. Bianchi, and L. T. Mainardi, "Advanced spectral methods for detecting dynamic behaviour," Autonomic Neuroscience: Basic and Clinical, vol. 90, no. 1-2, pp. 3-12, 2001.

[18] W. J. Kop, R. J. Verdino, J. S. Gottdiener, S. T. O’Leary, C. N. Bairey Merz, and D. S. Krantz, "Changes in heart rate and heart rate variability before ambulatory ischemic events," Journal of the American College of Cardiology, vol. 38, no. 3, pp. 742-749, 2001.

[19] B. D. Bertolet and C. J. Pepine, "The vascular endothelium as a key to understanding coronary spasm and syndrome X," Current Opinion in Cardiology, vol. 6, no. 4, pp. 496-502, 1991.

A. Smrdel received B.S., M.S., and Ph.D. degrees in computer and information science from the University of Ljubljana, Ljubljana, in 1997, 2000, and 2004, respectively. Between 1997 and 2000, he was a young researcher, and since 2001 he has been an Assistant in the Faculty of Computer and Information Science at the University of Ljubljana. His research interests include biomedical signal processing and other related topics.

F. Jager received a B.S. degree and an M.S. degree in electrical engineering from the University of Ljubljana in 1980 and 1984, respectively. In 1994, he received a Ph.D. degree in computer and information science from the University of Ljubljana. Currently, he is a Full Professor in the Faculty of Computer and Information Science at the University of Ljubljana, and a Research Affiliate at the Massachusetts Institute of Tech-

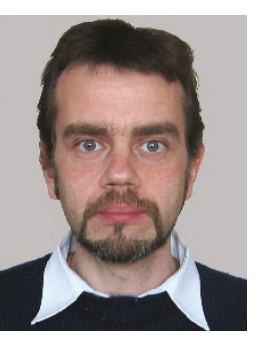
nology. His research interests include biomedical signal processing and medical imaging.

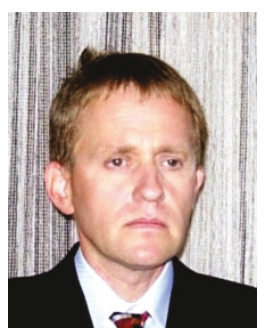

